\title{
Rate Dependent Constitutive Modelling of Laminated FRP Composites Degraded by Fire
}

\author{
R. J. Asaro, P. Krysl, B. Zhu, and W. T. Ramroth * \\ Department of Structural Engineering \\ University of California, San Diego \\ La Jolla, CA 92093
}

April 2003

\begin{abstract}
A constitutive theory is proposed for FRP laminated composite materials that is designed to account for both temperature and strain rate dependent response, such as would occur during, and after, exposure to the elevated temperatures due to fire. The theory is physically based, and in particular, is based on a kinematical framework fixed on the fabric laminates; full accounting of laminate reorientation and anisotropic response is thus achieved. The theory is numerically implemented and FEM analysis of compressive deformation of a sandwich panel, given as an example, demonstrates how common material failure modes such as kinking are naturally included in the theory and in analyses using the theory. The theory accounts for finite strains and thus extent of deformation is arbitrary. The theory and its numerical implementation are designed specifically to perform numerical analysis of structural response of FRP structures subject to fire degradation.
\end{abstract}

*Research supported by ONR 


\section{Introduction}

The single greatest impediment to the use of FRP composites in the design and construction of advanced ship systems and ship structures is their susceptibility to degradation due to exposure to the elevated temperatures caused by exposure to fire. On the one hand, FRP materials degrade through the direct loss of material by ablation and char formation, and on the other hand they degrade just by exposure to elevated temperatures in the range of say, $60^{\circ} \mathrm{C}<T<125^{\circ} \mathrm{C}$. Losses in material strength result from such exposures while the materials are at these temperatures and they undergo losses even after the materials are returned to ambient temperatures. Residual material properties fare better and for FRP material systems currently proposed for use for load bearing structures, residual properties can be maintained at near $100 \%$ even after exposures to temperatures of up to $200^{\circ} \mathrm{C}$. Some examples of material data are shown below. A constitutive framework is therefore required for describing the elastic-viscoplastic response of FRP materials to elevated temperatures that can be used to analyze the observations of materials behavior during laboratory testing and that can be used to perform full-scale structural analyses of structures subjected to fire. This paper is a first report on the development of such a constitutive framework.

The development described herein was guided by the dual needs of developing a theory that could embody the phenomenology of material loss as well as degradation in properties of intact material, yet be analytically tractable and computationally efficient to allow for engineering design. The material model would, in addition, have to be amenable to implementation along with the algorithms being co-developed for describing phase transformations as occur during material ablation. Topics such as ablation, char formation, and material loss will be described in other reports; here we confine our attention to the material theory describing time and temperature dependent elastic-plastic material response.

In many of its aspects our new theory borrows from the successful development of the theory for crystal plasticity, especially as laid out by Asaro and Rice (1977), Asaro and Needleman (1985), and Harren and Asaro (1989); specific citations in context are given where appropriate in the text. The theory will account fully for the anisotropy of FRP laminate elastic behavior as well as for the highly anisotropic inelastic response that occurs due to interlaminar shear. The latter process is strongly influenced by temperature. As noted above, there is the very real need for computational efficiency and 
this has led us to avoid constructing a discrete aggregate model, which while attractive in its ability to separately describe the roles of polymer matrix and fabric, would inevitably lead to a far more computationally expensive implementation that would render performing (many) engineering design simulations inviable. The framework for constructing such models, in fact, already exists as the need may arise and a particularly relevant foundation lies in the cell models recently proposed by Gu and Asaro (2002).

Of particular interest is the ability to describe common failure mechanisms that occur in FRP materials. Along with general elastic-viscoplastic deformation at elevated temperature these are seen to arise particularly as a result of compressive loading. One such failure mode is micro-buckling or kinking, which typically leads to rapid, often localized, degradation and fracture. We desire, therefore, a theory that naturally contains the occurrence of such phenomena. The model need also contain an accurate description of the temperature dependence of material stiffness and material resistance to interlaminar shear - these are accordingly key focuses of our development. The plan of the paper is as follows. We review some important features of time and temperature response of FRP composites exposed to elevated temperature in the next section. There we also describe key features of the mechanisms and kinematics of elastic and plastic deformation that will have to incorporated within a continuum theoretical framework. The constitutive theory is then presented in the next section. Some applications of the theory are made in Section 4 and conclusions are given in Section 5.

\section{Temperature Dependent FRP Behavior}

Figure 1 illustrates the in-situ temperature dependent properties of a typical vacuum bagged E-glass vinylester FRP composite. As noted, several properties are listed but as it happens they conveniently fall within a tight enough band that a single curve adequately represents their dependence on temperature.

It should be noted, however, that at temperatures above, say $50^{\circ} \mathrm{C}$ the properties are also somewhat dependent on time as well as temperature itself. The specific properties shown were recorded after approximately $30 \mathrm{~min}$ of exposure to temperature. Also shown in the figure are properties for two common core materials, viz. balsa wood and a PVC foam. For this particular material nearly all strength and stiffness is lost above about $125^{\circ} \mathrm{C}$. It is 


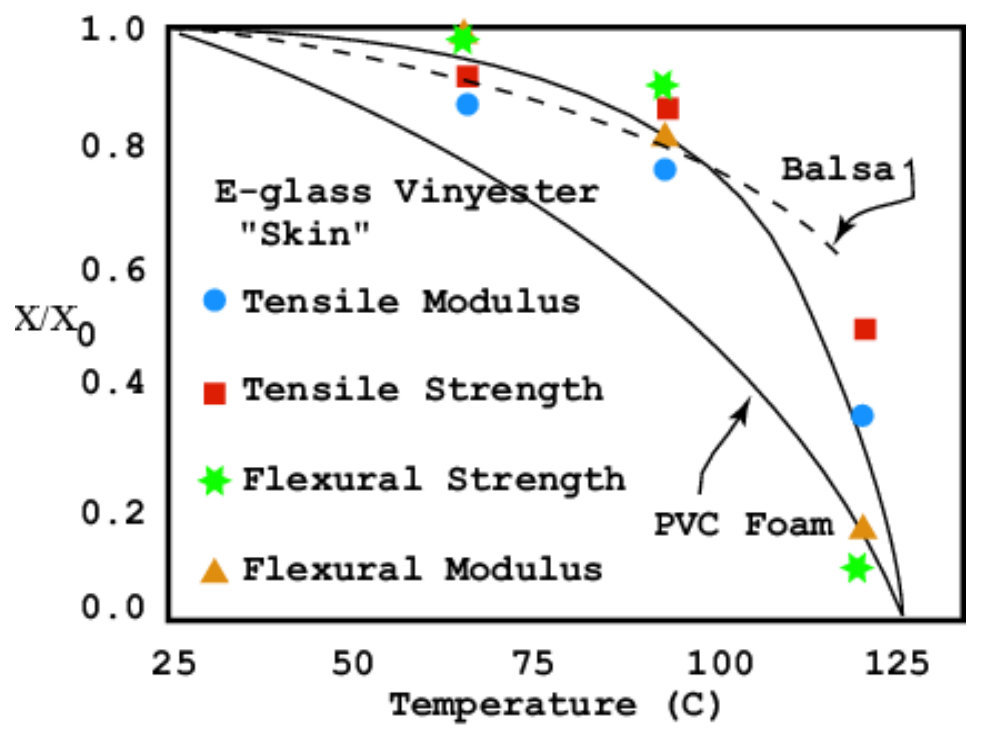

Figure 1: Temperature dependent properties

also important to note that the losses in material properties shown in Fig. 1 do not involve material ablation, i.e. do not involve material loss through combustion. Clearly the degradation shown in Fig. 1 must be attributed to a reduction in strength and/or stiffness of intact, but degraded material.

Property degradation comes about essentially due to a loss in the stiffness properties of the resin matrix and this process needs to be understood at more fundamental levels than it is at present. For the purposes of the present development, however, it is sufficient to calibrate moduli values to match the behavior shown. This is what is proposed as described below in Section 3 .

\section{The Constitutive Model}

\subsection{Nomenclature \& Conventions}

Standard notations are used throughout. Bold-faced symbols are used to denote vectors and higher order tensors, the order of which will be clear in context. Products are indicated with dots, which denote summation over repeated Latin indices, and products without dots are dyadic products. Latin indices range from one to the number of spatial dimensions (usually three), 
and repeated Latin indices are always summed. Inverses, transposes, and transpose inverses are denoted with a superscripted $-1, T$, and $-T$, respectively, and superposed dots indicate differentiation with respect to time, t. For instance,

$$
\begin{array}{cl}
\mathbf{A} \cdot \mathbf{B}=A_{i k} B_{k j} \mathbf{b}_{i} \mathbf{b}_{j}, & \mathbf{A}: \mathbf{B}=A_{i j} B_{j i}, \\
\mathbf{c d}=c_{i} d_{j} \mathbf{b}_{i} \mathbf{b}_{j}, & \mathbf{c} \cdot \mathbf{d}=c_{i} d_{i}, \\
\mathbf{H}: \mathbf{A}=H_{i j k l} A_{l k} \mathbf{b}_{i} \mathbf{b}_{j}, & \mathbf{A}: \mathbf{H}=A_{k l} H_{l k i j} \mathbf{b}_{i} \mathbf{b}_{j}, \\
\dot{\mathbf{B}}=\frac{\partial B_{i j}}{\partial t} \mathbf{b}_{i} \mathbf{b}_{j}, & \mathbf{B} \cdot \mathbf{c}=B_{i k} c_{k} \mathbf{b}_{i},
\end{array}
$$

where the base vectors $\mathbf{b}$ are Cartesian and independent of time. Greek indices are slip system identifiers ranging from one to two for the case of an orthotropic laminate such as considered herein and as explained below.

\subsection{The Laminate Model}

We consider the FRP material to be composed of an essentially orthotropic laminate, and to contain a sufficient number of plies so that homogenization is a reasonable way to describe the material behavior. The principal directions of the fibers are described by a set of mutually orthogonal unit base vectors, a, as depicted in Fig.2. The resulting orthotropic

elastic response of the laminated composite will, thus, be fixed on, and described by these vectors. The material can also deform via slipping in the plane of the laminate, i.e. via interlaminar shear, and this slipping is confined to this interlaminar plane. Slipping is possible in all directions in the plane, but not necessarily with equal ease. We thus introduce two slip systems aligned with the slip directions $\mathbf{s}_{1}$ and $\mathbf{s}_{2}$. The normal to the laminate plane is $\mathbf{m}$, and clearly $\mathbf{s}_{1} \cdot \mathbf{m}=0$ and likewise $\mathbf{s}_{2} \cdot \mathbf{m}=0$. It may well be natural, but not necessary, to take $\mathbf{s}_{1}$ and $\mathbf{s}_{2}$ to be orthogonal, i.e. $\mathbf{s}_{1} \cdot \mathbf{s}_{2}=0$, but note that due to elastic distortions they may not remain so during deformation. These vectors will be called $\mathbf{s}_{1}^{*}, \mathbf{s}_{2}^{*}$, and $\mathbf{m}^{*}$ in the deformed state, but since $\mathrm{m}^{*}$ is to be the normal to the slip plane, i.e. the plane of the laminate, it will always be the case that $\mathbf{s}_{1}^{*} \cdot \mathbf{m}^{*}=0$ and $\mathbf{s}_{2}^{*} \cdot \mathbf{m}^{*}=0$ as shown to be naturally described by our expressions for the kinematics of laminate deformation. In fact, it is possible to take the slip system vectors to be coincident with the laminate base vectors, a and insure that they are convected so that the above stated orthogonality is preserved; there is no 


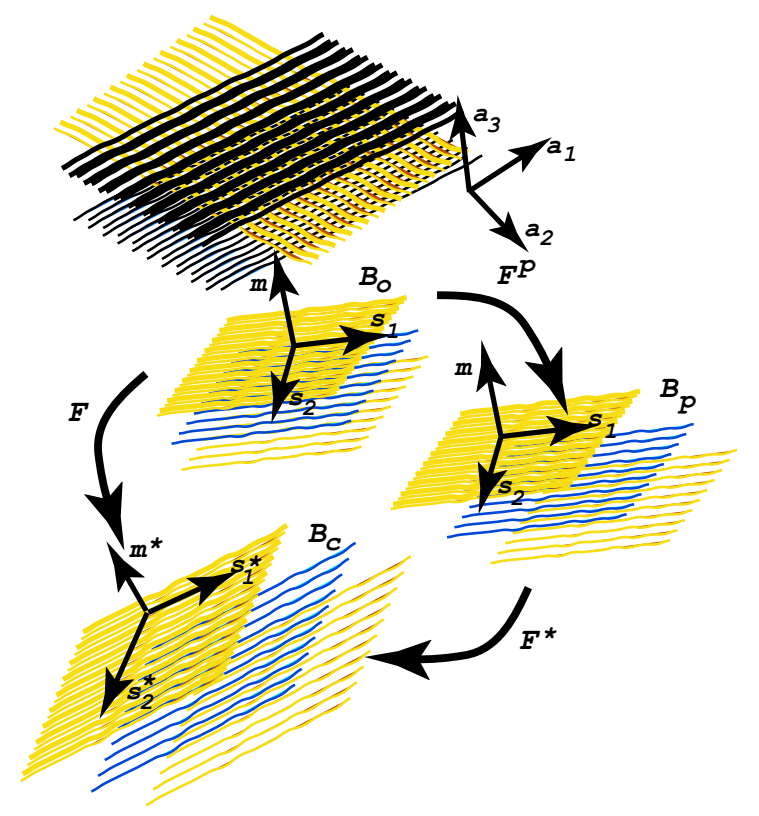

Figure 2: Kinematics

need to do this however. Even though both slip systems have the same slip plane normal, i.e. $\mathbf{m}$, it will be convenient for symmetry of expression to refer to $\mathbf{m}_{1}$ and $\mathbf{m}_{2}$ in the expressions below. This makes it easier as well to establish correlations with the body of theory for crystal plasticity. Figure 2 illustrates the total deformation from the reference configuration, $\mathcal{B}_{0}$, to occur in two stages producing a viscoplastic and an elastic deformation, respectively. The viscoplastic deformation occurs by the flow of the material through the framework, i.e. the lattice, of the laminate via the interlaminar shears. The spatial velocity gradient of this plastic flow is thus written as

$$
\dot{\mathbf{F}}^{p} \cdot \mathbf{F}^{p-1}=\sum_{1}^{2} \dot{\gamma}_{\alpha} \mathbf{s}_{\alpha} \mathbf{m}_{\alpha},
$$

where $\mathbf{F}^{p}$ is the plastic deformation gradient and $\dot{\gamma}_{\alpha}$ is the rate of shearing on the $\alpha_{t h}$ slip system. The value of $\mathbf{F}^{p}$ is obtained by the path dependent integration of (1). This process produces an intermediate configuration, $\mathcal{B}_{p}$. Next the current, i.e. deformed, configuration $\mathcal{B}_{c}$ is reached from $\mathcal{B}_{p}$ by elastically distorting and rigidly rotating the laminate (i.e. lattice) along with material, i.e. fabric and matrix, embedded on it. This second step of 
deformation is described by the elastic deformation gradient, $\mathbf{F}^{*}$. Hence the decomposition of $\mathbf{F}$,

$$
\mathbf{F}=\mathbf{F}^{*} \cdot \mathbf{F}^{p}
$$

is obtained, where $\mathbf{F}$ is the total elastic plus plastic deformation. This decomposition was introduced in this context for crystal plasticity by Asaro and Rice (1977) and for phenomenological plasticity by Lee (1969).

The driving force for slip on the $\alpha_{t h}$ system is taken to be primarily caused by the resolved shear stress, $\tau_{\alpha}$ on that system. This is written as,

$$
\tau_{\alpha}=\mathrm{m}_{\alpha}^{*} \cdot \tau \cdot \mathrm{s}_{\alpha}^{*}, \mathrm{~s}_{\alpha}^{*}=\mathrm{F}^{*} \cdot \mathrm{s}_{\alpha}, \mathrm{m}_{\alpha}^{*}=\mathrm{m}_{\alpha} \cdot \mathrm{F}^{*-1}, \quad \tau=\mathrm{J} \sigma,
$$

where $\mathbf{J}=\operatorname{det}\{\mathbf{F}\}$ is the Jacobian, $\boldsymbol{\tau}$ is the Kirchhoff stress, and $\boldsymbol{\sigma}$ is the Cauchy stress (i.e. true) stress. Here, in the current state (i.e. deformed state), $\mathbf{s}_{\alpha}^{*}$ and $\mathbf{m}_{\alpha}^{*}$ are respectively along the $\alpha_{t h}$ slip direction and the slip plane normal. Note that $\mathbf{s}_{1}$ and $\mathbf{s}_{2}$ are made to convect with the lattice framework whereas $\mathbf{m}$ is the reciprocal base vector that remains normal to the plane that contains both $\mathbf{s}_{1}$ and $\mathbf{s}_{2}$. This definition of $\tau_{\alpha}$ as discussed by Asaro (1983) is used because it makes $\tau_{\alpha}$ conjugate to $\dot{\gamma}_{\alpha}$, i.e. $\sum_{\alpha=1}^{2} \tau_{\alpha} \dot{\gamma}_{\alpha}$ is precisely the plastic dissipation rate per unit reference volume. On the other hand it is entirely possible that the shear resistance of interlaminar zones is sensitive to the normal stress that acts on the plane, the shear rate would depend on the stress component, $\tau_{m m}=\mathbf{m}^{*} \cdot \boldsymbol{\tau} \cdot \mathbf{m}^{*}$. In this case a more general load parameter might be prescribed for the $\alpha_{t h}$ slip system, viz. $\hat{\tau}_{\alpha}=\left\|\tau_{\alpha}\right\|+$ $\beta \tau_{m m}$, but where $\hat{\tau} \geq 0$. The parameter $\beta$ represents are frictional resistance due to the compaction of fabric due to normal compressive stresses. Note that as shown by Asaro and Rice (1977) and then again by Dao and Asaro (1996) the appearance of such terms in the loading parameter represents a deviation from the law of normality which, in turn, has implications for localized deformation.

The constitutive description of the plasticity on each slip system is cast in terms of the resolved shear stress on that system and on the current slip rate on that system as,

$$
\dot{\gamma}_{\alpha}=\dot{\gamma}_{0} \operatorname{sgn}\left\{\tau_{\alpha}\right\}\left\{\left\|\frac{\tau_{\alpha}}{g_{\alpha}}\right\|\right\}^{1 / m},
$$

where $\tau_{\alpha}$ is the current value of the resolved shear stress and $g_{\alpha}$ is the current value of the slip system hardness. Note that when frictional effects are important $\tau_{\alpha}$ should be replaced by $\hat{\tau}_{\alpha}$ in (4). In (4), $m$ is the strain rate 
sensitivity exponent, $\dot{\gamma}_{0}$ is a reference shear strain rate, and $\operatorname{sgn}\{\bullet\}$ means the sign of $\{\bullet\}$. Note that as $m \rightarrow 0$ rate independent behavior is achieved, and that in that limit $g_{\alpha}$ corresponds to the slip system strength, $\tau_{\alpha}$ at least in absolute value. This limit is unlikely for the polymer resin systems of interest here. Also, for creep-like behavior we expect $0.15 \leq m \leq 1.0$, whereas for viscoplastic response $m \leq 0.1$.

In general, we expect the possibility that there may be hardening of the interlaminar layers following slip and this is described by a path dependent evolution equation of the form,

$$
\dot{g}_{\alpha}=\sum_{\beta=1}^{2} h_{\alpha \beta}\left(\gamma_{a}\right)\left\|\dot{\gamma}_{\beta}\right\|, \quad \gamma_{a}=\int_{0}^{t} \sum_{\alpha}\left\|\dot{\gamma}_{\alpha}\right\| d t
$$

where $h_{\alpha \beta}$ is a hardening matrix of (non-negative) hardening moduli and $\gamma_{a}$ is the accumulated sum of the slips. The initial conditions for this evolution are specified as $g_{\alpha}\left(\dot{\gamma}_{\alpha}=0\right)=g_{0}$ and the form of $h_{\alpha \beta}$ is,

$$
h_{\alpha \beta}\left(\gamma_{a}\right)=g \prime\left(\gamma_{a}\right) q_{\alpha \beta},
$$

where the prime denotes differentiation with respect to $\gamma_{a}$, and $q_{\alpha \beta}$ is a matrix that describes the cross hardening between the two slipping directions. A possible, and quite general form for $g\left(\gamma_{a}\right)$ is,

$$
g\left(\gamma_{a}\right)=g_{0}+h_{\infty} \gamma_{a}+\left(g_{\infty}-g_{0}\right) \tanh \left\{\frac{\left(h_{0}-h_{\infty}\right)}{\left(g_{\infty}-g_{0}\right)}\right\} .
$$

If the laminate is subjected to a monotonically increasing shear strain $\gamma>0$ along one direction, then $\gamma=\gamma_{a}$ and the relation $g=g\left(\gamma_{a}\right)$ can be interpreted as being the relation of hardness vs. shear strain for that slip system. Also, in (7), $g_{0}=g\left(\gamma_{a}=0\right), h_{0}=g \prime\left(\gamma_{a}=0\right)$, and $h_{\infty}=g \prime\left(\gamma_{a} \rightarrow \infty\right)$. If $h_{\infty} \equiv 0$, then $g_{\infty}=g\left(\gamma_{a} \rightarrow \infty\right)$. At present there is little data to guide the calibration of hardening laws such as (7), but its flexibility should provide the ability to do so.

The description of the laminate's constitutive response is completed with a specification of its elasticity, which is expressed in terms of the Green strain of the fabric framework, viz. $\mathbf{E}^{*}=(1 / 2)\left(\mathbf{F}^{* T} \cdot \mathbf{F}^{*}-\mathbf{I}\right)$ and the laminate framework-based second Piola-Kirchhoff stress, $\mathbf{S}^{*}=\mathbf{F}^{-1} \cdot \boldsymbol{\tau} \cdot \mathbf{F}^{*-T}$, where $\mathbf{I}$ is the second-order identity tensor. For anisotropic elastic solids the elastic response may be written as $S_{i j}^{*}=\left(\partial \Phi / \partial E_{i j}^{*}\right)$, where $\mathbf{S}^{*}=S_{i j}^{*} \mathbf{a}_{i} \mathbf{a}_{j}, \mathbf{E}^{*}=$ 
$E_{i j}^{*} \mathbf{a}_{i} \mathbf{a}_{j}$, and $\Phi=\Phi\left(E_{i j}^{*}\right)$ is the free energy of the fabric framework per unit reference volume. Alternatively, in rate form, we have

$$
\dot{\mathbf{S}}^{*}=\mathbf{K}: \dot{\mathbf{E}}^{*}, \quad \mathbf{K}=K_{i j k l} \mathbf{a}_{i} \mathbf{a}_{j} \mathbf{a}_{k} \mathbf{a}_{l}, \quad K_{i j k l}=\frac{\partial^{2} \Phi}{\partial E_{i j}^{*} \partial E_{k l}^{*}}
$$

On the other hand (8) can, and typically will, be constructed from a laminate theory based on discrete analysis of the layering of thin plies.

\subsection{Additional Kinematical Perspective}

The decomposition of (2) results in the total velocity gradient taking the form,

$$
\dot{\mathbf{F}} \cdot \mathbf{F}^{-1}=\dot{\mathbf{F}}^{*} \cdot \mathbf{F}^{*-1}+\mathbf{F}^{*} \cdot \sum_{\alpha=1}^{2} \dot{\gamma}_{\alpha} \mathbf{s}_{\alpha} \mathbf{m}_{\alpha} \cdot \dot{\mathbf{F}}^{*-1} .
$$

By forming symmetric and antisymmetric parts of the above velocity gradient we obtain the decomposition of the rate of deformation and the spin rates, viz.

$$
\mathrm{D}=\mathrm{D}^{*}+\mathrm{D}^{p} \quad \Omega=\Omega^{*}+\Omega^{p}
$$

where

$$
\mathrm{D}^{p}=\sum_{\alpha=1}^{2} \mathrm{P}_{\alpha} \dot{\gamma}_{\alpha} \quad \Omega^{p}=\sum_{\alpha=1}^{2} \mathrm{~W}_{\alpha} \dot{\gamma}_{\alpha}
$$

In (11), the symmetric and antisymmetric tensors $\mathbf{P}_{\alpha}$ and $\mathbf{W}_{\alpha}$ are given as,

$$
\mathbf{P}_{\alpha}=\frac{1}{2}\left\{\mathbf{s}_{\alpha}^{*} \mathbf{m}_{\alpha}^{*}+\mathbf{m}_{\alpha}^{*} \mathbf{s}_{\alpha}^{*}\right\} \quad \mathbf{W}_{\alpha}=\frac{1}{2}\left\{\mathbf{s}_{\alpha}^{*} \mathbf{m}_{\alpha}^{*}-\mathbf{m}_{\alpha}^{*} \mathbf{s}_{\alpha}^{*}\right\} .
$$

The elastic parts of $\mathbf{D}$ and $\mathbf{W}$, viz. $\mathbf{D}^{*}$ and $\mathbf{W}^{*}$ are formed by taking the symmetric and antisymmetric parts of (9). Note that the interpretations of how $\mathbf{s}$ and $\mathbf{m}$ convect with the laminate framework, as introduced in (3), are a natural outcome of the kinematical scheme shown in Fig. 1.

\subsection{Final Constitutive Forms}

The description of the single crystal's constitutive response is completed with a specification of its elasticity, which is expressed in terms of the Green strain of the framework, $\mathbf{E}^{*}$, as given in (8). Having the description of this 
response the entire constitutive theory can be expressed in terms of the Green strain, $\mathbf{E}=1 / 2\left\{\mathbf{F}^{T} \cdot \mathbf{F}-\mathbf{I}\right\}$ and the second Piola-Kirchhoff stress, $\mathbf{S}=$ $\mathbf{F}^{-1} \cdot \boldsymbol{\tau} \cdot \mathbf{F}^{-\boldsymbol{T}}$. Straightforward tensor manipulations of the above relations yield the governing constitutive rate form,

$$
\dot{\mathbf{S}}=\mathbf{L}: \dot{\mathbf{E}}-\sum_{\alpha=1}^{2} \dot{\gamma}_{\alpha} \mathbf{X}_{\alpha}
$$

where

$$
L_{i j r n}=F_{i k}^{p-1} F_{j l}^{p-1} K_{k l p q} F_{r p}^{p-1} F_{n q}^{p-1}
$$

and where

$$
\mathbf{L}=L_{i j k l} \mathbf{a}_{i} \mathbf{a}_{j} \mathbf{a}_{k} \mathbf{a}_{l}, \quad \mathbf{F}^{p-1}=F_{i j}^{p-1} \mathbf{a}_{i} \mathbf{a}_{j}
$$

In $(13)$

$$
\mathbf{X}_{\alpha}=\mathbf{F}^{p-1} \cdot\left\{\mathbf{K}: \mathbf{A}_{\alpha}+2 \mathbf{B}_{\alpha}\right\} \cdot \mathbf{F}^{p-1}
$$

with

$$
\mathbf{A}_{\alpha}=\operatorname{sym}\left\{\mathbf{F}^{* T} \cdot \mathbf{F}^{*} \cdot \mathbf{s}_{\alpha} \mathbf{m}_{\alpha}\right\}, \quad \mathbf{B}_{\alpha}=\operatorname{sym}\left\{\mathbf{s}_{\alpha} \mathbf{m}_{\alpha} \cdot \mathbf{S}^{*}\right\} .
$$

Finally, we note that it is useful to express the constitutive relations in terms of the nominal stress, $\mathbf{n}$ and its conjugate, $\mathbf{F}$ itself. The transformation is also straightforward since $\mathbf{n}=\mathbf{F}^{-1} \cdot \boldsymbol{\tau}=\mathbf{S} \cdot \mathbf{F}^{\boldsymbol{T}}$ and consequently $\dot{\mathbf{n}}=$ $\dot{\mathbf{S}} \cdot \mathbf{F}^{T}+\mathbf{S} \cdot \dot{\mathbf{F}}^{T}$, which then yields

$$
\dot{\mathbf{n}}=\mathbf{M}: \dot{\mathbf{F}}-\sum_{\alpha=1}^{2} \dot{\gamma}_{\alpha} \mathbf{Y}_{\alpha}
$$

with

$$
\begin{aligned}
M_{i j k l} & =F_{i a}^{p-1} F_{j b}^{*} K_{a b c d} F_{k c}^{p-1} F_{i d}^{*}+S_{i k} \delta_{j l} \\
\mathbf{F}^{*} & =F_{i j}^{*} \mathbf{a}_{i} \mathbf{a}_{j}, \quad \mathbf{S}=S_{i j} \mathbf{a}_{i} \mathbf{a}_{j} \\
\mathbf{I} & =\delta_{i j} \mathbf{a}_{i} \mathbf{a}_{j}, \quad \mathbf{M}=M_{i j k l} \mathbf{a}_{i} \mathbf{a}_{j} \mathbf{a}_{k} \mathbf{a}_{l},
\end{aligned}
$$

with

$$
\mathbf{Y}_{\alpha}=\mathbf{X}_{\alpha} \cdot \mathbf{F}^{T}
$$




\subsection{Calibration of the constitutive model}

To account for the loss in material stiffness at elevated temperatures we will take, as a first step, the curve shown in Fig. 1 to be a master degradation function for all components of moduli. This means that in (8) $K_{i j k l} \leftarrow$ $f(T) K_{i j k l}$, where $f(T)$ is the monotonically decreasing function shown in the figure. It should be possible to improve on this simple representation of stiffness degradation with further experimental study of the effect of temperature on anisotropic stiffness. Losses in shear strength are handled, again as a first step in our modelling, by replacing $g\left(\gamma_{a}\right)$ in (4) with $\eta\left(\gamma_{a}\right)$, where $\eta=r(T) g\left(\gamma_{a}\right)$. The function $r(T)$ is to be determined by experimental documentation of the effect of temperature on the resistance to interlaminar shear. Thus, the shearing rates are calculated from,

$$
\dot{\gamma}_{\alpha}=\dot{\gamma}_{0} \operatorname{sgn}\left\{\tau_{\alpha}\right\}\left\{\left\|\frac{\tau_{\alpha}}{\eta_{\alpha}}\right\|\right\}^{1 / m},
$$

In the examples shown in the next section $r(T)$ is taken to be of the same general monotonically decreasing form as $f(T)$.

In general, orthotropic elastic symmetry is presumed although the materials will often possess transverse isotropy. Thus the elastic constants will have components, when phrased on the orthotropic axes of the form,

$$
\mathbf{K}=K_{i j k l} \mathbf{a}_{i} \mathbf{a}_{j} \mathbf{a}_{k} \mathbf{a}_{l} .
$$

If the common convention of index contraction is used, i.e. $11 \leftrightharpoons 1,22 \leftrightharpoons 2$, $33 \leftrightharpoons 3,23,32 \leftrightharpoons 4,13,31 \leftrightharpoons 5$, and $12,21 \leftrightharpoons 6$, the matrix of components becomes,

$$
\left\{\begin{array}{cccccc}
K_{11} & K_{12} & K_{13} & 0 & 0 & 0 \\
K_{12} & K_{22} & K_{23} & 0 & 0 & 0 \\
K_{13} & K_{23} & K_{33} & 0 & 0 & 0 \\
0 & 0 & 0 & K_{44} & 0 & 0 \\
0 & 0 & 0 & 0 & K_{55} & 0 \\
0 & 0 & 0 & 0 & 0 & K_{66}
\end{array}\right\}
$$

the elements of this stiffness matrix may be formed directly from experimental measurement of the elastic moduli, or from standard laminate theory. 


\section{Applications}

We consider below the compressive deformation of a sandwich panel, as an example application of the constitutive model. The example is specifically chosen to illustrate the ability of the model to predict failure modes such as kinking. We note, as discussed above, that with degradation due to elevated temperatures, interlaminar shear, i.e. slip, is induced and this will lead to an early onset of failure modes such as kinking. Of course, other failure modes such as skin wrinkling are also likely to be triggered and these will compete with failure modes such as structural buckling and kinking. Our constitutive and numerical framework is capable of describing these modes, as they compete, en toto. In the present example, however, we will focus on kinking as described below.

\subsection{Kinking of sandwich composite skin}

\subsubsection{Problem description}

The sandwich is taken to be symmetric and consists of a relatively compliant core (balsa wood) and skins of symmetric lay-up with respect to the midplane of the sandwich, held together by an adhesive layer. In the particular case examined, the core is $50 \mathrm{~mm}$ thick, and the skins are of $1.75 \mathrm{~mm}$ thickness. Figure 3 shows a schematic view and the finite element mesh and also helps to explain the model. The specimen is further taken to be $100 \mathrm{~mm}$ tall, and is modeled with plane-strain constraint in the $y$ direction (into the plane of the drawing) with one layer of solid hexahedral 8-noded finite elements. Only one half is discretized as symmetry is assumed. It is noted here, and below, that the specimen is purposely taken to be "stubby" so as to effectively preclude the appearance of structural buckling. This is done so that the appearance of kinking modes, that are inherent in the theory, would be highlighted. As discussed below, when more slender specimens are analyzed, kinking modes would compete with structural buckling modes and indeed a typical specimen, or structure, would display combinations of such modes.

The core is modeled as an isotropic elastic material. The constitutive model outlined above is used for the skin of the composite with slip plane normal parallel in the reference configuration to the surface of the skin. There is, however, a geometrical imperfection in the slip plane normal at about the midpoint of the panel. In particular, between $z=45 \mathrm{~mm}$ and $z=45 \mathrm{~mm}$, 
the direction of the normal is assumed to have components $\widetilde{N}_{x}=1, \widetilde{N}_{y}=0$ and

$$
\widetilde{N}_{z}=-\frac{\pi}{10} \sin \left(\frac{\pi}{20}(z-35)\right) \cos \left(\frac{\pi}{20}(z-35)\right) .
$$

(The actual slip plane normal is normalized to unit length, $N_{i}=\widetilde{N}_{i} /\|\widetilde{\mathbf{N}}\|$.) When the specimen is subjected to compression in the $z$ direction, we therefore expect to see $\operatorname{kink}(\mathrm{s})$ formed in the skin(s) of the sandwich composite. The core and skins are held together by a single layer of thin elements, designed to replicate the adhesive layer. These elements have properties designed to simulate the debonding of the skin once the maximum strength of the adhesive (or tensile strength of the core) is reached. In particular, when the positive principal stress value exceeds a given limit, the element's internal stress is assigned a zero value, thus effectively deactivating the quadrature point. This limiting decohesive stress is $\sigma_{\max }=1 \mathrm{MPa}$. Alternate, and more elaborate, cohesive models can be implemented, see for example Varias, O'Dowd, Asaro and Shih (1990); and also Xu and Needleman (1996).

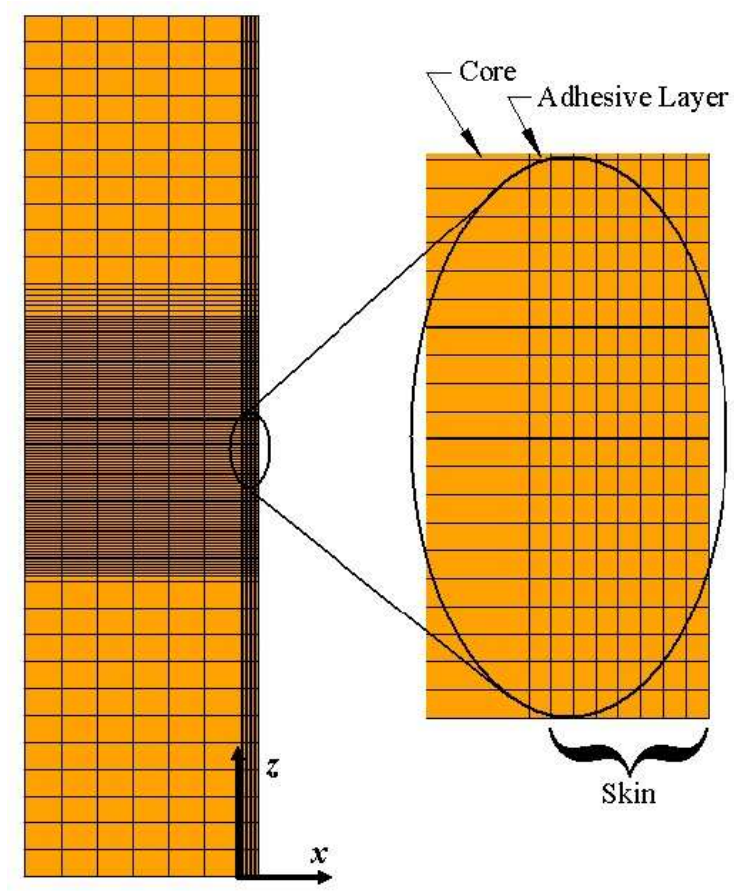

Figure 3: View of the overall geometry and the zoom-in of the imperfection area. 


\subsubsection{Material Parameters}

For computational economy, in this example, only the center part $(35 \mathrm{~mm}<$ $z<65 \mathrm{~mm}$ ) of the specimen skin is elasto-viscoplastic; other parts of the skin are assumed to remain (hyper-) elastic. The constitutive parameters of the skin material are as follows: elastic constants are $c_{11}=204000 \mathrm{MPa}$, $c_{12}=68000 \mathrm{MPa}, c_{44}=68000 \mathrm{MPa}$, (implying isotropy), isotropic or Taylor hardening parameter $q=1$, initial hardening rate $h_{0}=1$, reference shear strain rate $\gamma_{0}=300$, initial flow stress $g_{0}=27 \mathrm{MPa}$, saturation strength $g_{\text {inf }}=30 \mathrm{MPa}$, strain-rate sensitivity exponent $m_{\text {srs }}=0.05$, material density $\rho=1.6 \times 10^{-9}$ ton $\mathrm{m}^{-3}$. Note that it has been assumed that the skins are elastically isotropic for the example shown.

The core is elastic, and the material parameters are as follows: Young's modulus $E=17000 \mathrm{MPa}$, Poisson's ratio $\nu=0$, material density $\rho=$ $0.6 \times 10^{-9}$ ton $\mathrm{m}^{-3}$. The adhesive layer elements are assigned a tensile cut-off strength of $1 \mathrm{MPa}$.

\subsubsection{Initial and Displacement Boundary Condition}

The top surface is made to move downwards at $100 \mathrm{~mm} / \mathrm{s}$, and the bottom surface of the specimen is fixed in the $z$ direction. Therefore, the initial condition is a linearly interpolated velocity distribution in the $z$ direction. The plane-strain constraint is applied in the $y$ direction, and the skins at the top and bottom portions of the model are prevented from moving in the $x$ direction to prevent global buckling mode of failure. We note that in general global buckling is a failure mode that competes with kinking. We preclude it here because we are more interested at this point at examining the phenomenology of materials, rather than structural, failure modes.

\subsubsection{Results}

The results are reported in several ways. In particular, the resolved shear stresses and plastic slip strains are reported at nodes along mesh line through the thickness of the composite skin, viz. at $z=50 \mathrm{~mm}$, which is shown in Figure 4. There are nine (9) nodes on the plane. The first node on the line is at the interface between the core and the adhesive layer. The second lies between the adhesive layer and the skin, and the ninth is at the surface of the skin.

Figure 5 shows the nominal load vs. axial displacement for this example 




Figure 4: Sampling line drawn next to the deformed geometry with the contours of the slip strains.

calculation. Note the behavior is quite linear up to the point where slip begins as noted by the contours shown in Figure 6, discussed next. After yielding, and eventually the development of a kinking mode, the load drops rapidly. We note, however, that there is no overall "spring back" as the specimen is under displacement control.

Figure 6 shows the evolution of the plastic slip strain $\gamma_{2}$ on a series of snapshots from the time history, namely snapshots 198, 208, 218, 228, and 238 (238 is the last). These points are marked on Figure 5. As noted, Figure 6 illustrates the development of the inelastic deformation. The color range of the slip strain is fixed as $\langle-0.05 ; 0.05\rangle$. Figure 7 displays the corresponding series of images for the resolved shear stress for time snapshots 198, 208, 
218,228 , and 238. We note some interesting trends, namely that after general yielding in the imperfect region, and following kink formation, there is a drop off of the magnitude of the resolved shear stress, $\tau_{2}$. Figure 8 shows the curves of the time evolution of the resolved shear stress at the nodes along the sampling line. Figure 9 shows the corresponding evolution of the plastic slip strains at the nodes along the sampling line.

Figure 10 illustrates how the axial stress evolves along the fibers at positions along the sampling line at $z=50 \mathrm{~mm}$. As expected, the stresses are compressive at first during what is essentially a more-or-less uniform compression. Following the onset of kinking, and then full kinking, the stresses start to adjust to reflect the bending of the skin. This type of phenomenology is typical of kinking and the resulting tensile stresses promote fracture and thus failure of the skin. On the other hand, there are tensile stresses developed on the skin-core interface, and these will induce rupture of the adhesive layer (de-bonding of the skin from the core).

Finally, Figure 11 illustrates the $\sigma_{x x}$ stress in the adhesive layer at the $50 \mathrm{~mm}$ sampling line, as well as $2 \mathrm{~mm}$ below that sampling line. It is clear that at the location $2 \mathrm{~mm}$ below the sampling line, the composite skin is pulling away from the core. The tensile stress in the $\mathrm{x}$ direction builds until it reaches the adhesive layer strength of $1 \mathrm{MPa}$. The stress then drops to zero, indicating that debonding has occurred. The stress at the sampling line, on the other hand, initially is in compression. This couple at the adhesive layer balances the couple formed in the skin due to the eccentricity at the kink. The compression force at the sampling line increases up until the point of debonding in the tensile region, at which point the resisting couple in the adhesive layer shifts upward, resulting in its quick reversal. This force immediately rises to the $1 \mathrm{MPa}$ tensile limit and then itself debonds. As can be seen in the figure, debonding begins at a stage in the loading history proceeding the load drop in the record of Figure 5. It tends to propagate quickly once begun as the specimen begins to undergo compressive failure.

\section{Summary and Future Work}

The approach taken here is capable of describing failure due to a wide variety of failure modes, that themselves occur over a wide variety of physical size scales. Such modes include structural buckling, skin wrinkling (that leads, in turn, to skin delamination), and material failures such as the kinking we 


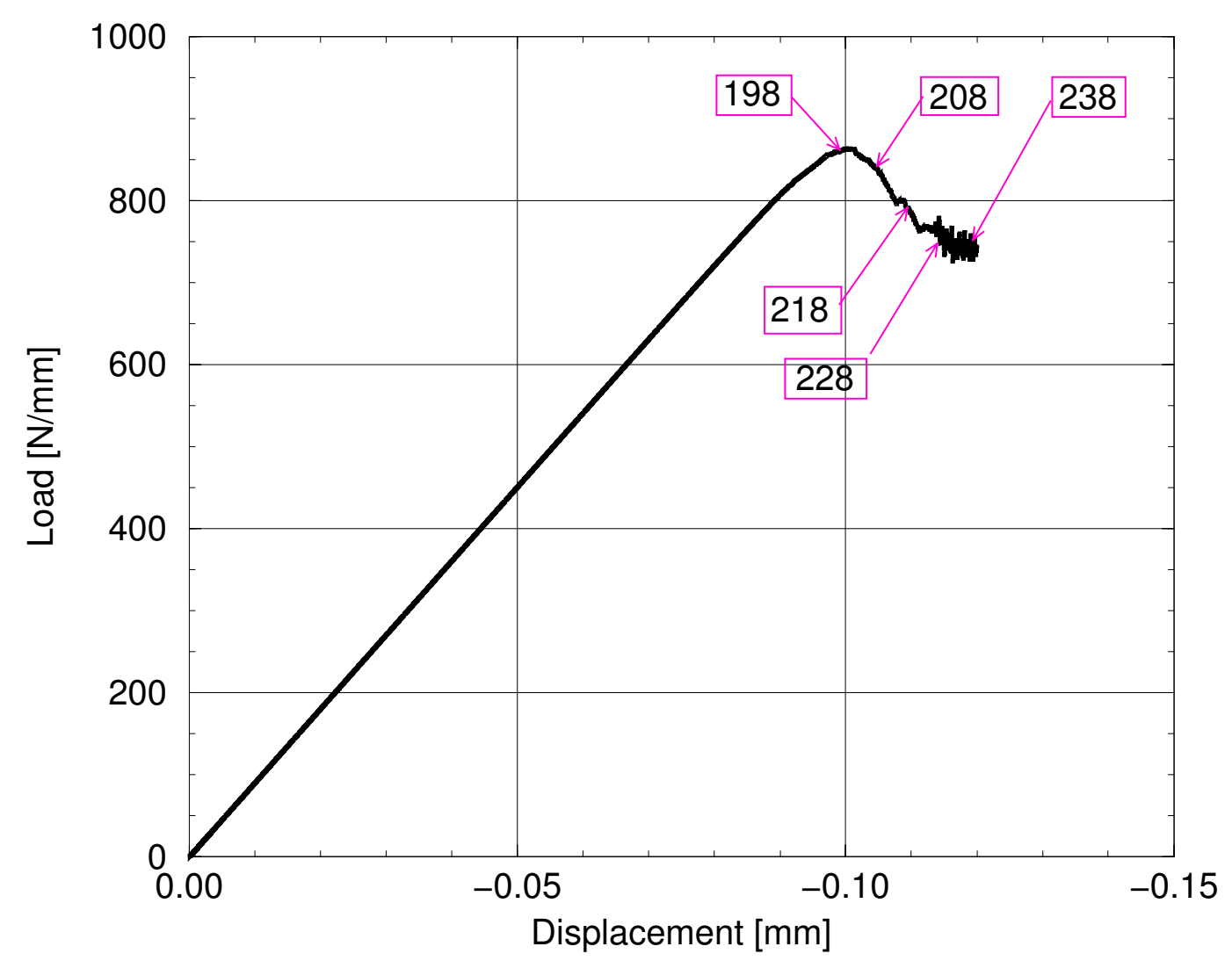

Figure 5: Load vs. displacement along $z$-direction

have explore in the example described above. We note, again, that in that example we purposely precluded structural modes by making the sandwich specimen suitably "stubby". This was done since our expressed aim was to demonstrate the ability of the constitutive theory to predict, and quantitatively describe, the onset and progression of material failures. Had we chosen a more slender geometry, and one more typical of a structural member, we would undoubtedly observed structural modes preceeding the kinking like modes described in the above example.

It should also be noted that the material properties used are those representative of "intact", essentially damage-free material. In the case of fire, and consequent material degredation, we expect to observe transitions in failure modes along with the earlier onset of failure modes that would have occurred only after attaining the anticipated - and designed for!-loads. It is 
precisely this sort of phenomenology that our approach is designed to handle in a natural and seamless fashion. That is, we developed herein an approach that does not require ad hoc input from extraneous failure models as postprocessing but rather naturally contains a physically based description of the entire gamut of failure modes. The theory we developed here also provides for a clear, tractable path to calibration.

The approach taken above to describe the time and temperature response has, however, been approximate in that it is based on a homogenized treatment of what is actually a discrete interaction between the constituents of FRP composites. It is obvious that much more work is required aimed at documenting the behavior of such composite materials that are subject to the elevated temperatures due to fire. It is also clear that the development of more physically based models for the temperature dependence will benefit from the co-development of micro-mechanical models for the interaction that takes place between laminates and the polymer resin matrices comprising the composites. As noted in the Introduction, the recently developed cell model of $\mathrm{Gu}$ and Asaro (2002) may represent a particularly good point of departure for such models as they have formulated an aggregate model that accounts for the discrete distribution of distinctly different phases within a coherent composite. Finite strains and rate dependent behavior are accounted for in what amounts to something more than just a self-consistent scheme. In fact in the their approach, both traction continuity and displacement continuity are prescribed between all sub-cells, that is distinct phases, of the composite. We are currently pursuing the refined development of such models and correlated experiments aimed at documenting the effects of temperature on the full 3D material response. There is, however, the need to retain computational tractability vis-á-vis the goal of performing engineering design computations. Thus whereas the micro-mechanically based models will be of great use in exploring complex phenomena and understanding failure, they are also likely to be used to guide the development of more accurate, but tractable, models of the type presented above.

As a final note, the authors have developed a detailed model for the thermal profile development in FRP composite materials subject to incident heat fluxes, or elevated surface temperatures (Krysl, 2003). The model accounts for thermal degradation caused by both elevated temperatures and material loss due to pyrolysis. It is, therefore, natural to combine that ability with the constitutive framework described herein to provide a complete description of thermal profiles, material degradation, and structural degradation caused by 
fire. As an example, Figure 12 shows results for the temperature distribution that developed during a simulation of the thermal loading of a sandwich panel similar to the type analyzed above. In the example, material is actually lost through pyrolysis which produces a "char" that has minimal, and even vanishing, properties. The temperature profile is obviously nonuniform and thus would, with reference to Figure 1, cause a severe and nonuniform degradation in material properties. The thermal analyses, such as illustrated in Figure 12, coupled through the properties described by Figure 1 and the constitutive calibration described in Section 3.5, to the thermo-mechanical theory described here would provide a complete structural analysis framework.

\section{References}

Asaro, R. (1983) J. appl. Mech. 50 p. 921.

Asaro, R. and Needleman, A. (1985) Acta Metall. 27 p. 45.

Asaro, R. J. and Rice, J. R. (1977) J. Mech. Phys. Solids 25 p. 309.

Dao, Ming and Asaro, Robert J. (1996) Scripta Materialia 34 p. 1771.

Gu, Pei and Asaro, R. J. (2002) Modelling and Simulation in Materials Science and Engineering 10 p. 477.

Harren, S. V. and Asaro, R. (1989) J. Mech. Phys. Solids 37 p. 191.

Krysl, P., Ramroth, W. T., Stewart, L. K., and Asaro, R. J. (2003) Int. J. Numer. Meth. Engng, to be published.

Lee, E. H. (1969) J. appl. Mech. 36 p. 1.

Varias, A. G., O’Dowd, N. P., Asaro, R. J, and Shih, C. F. (1990) Materials Science and Engineering A126 p. 65.

Xu, X. P. and Needleman, A. (1996) International Journal of Fracture 74 p. 289-324. 

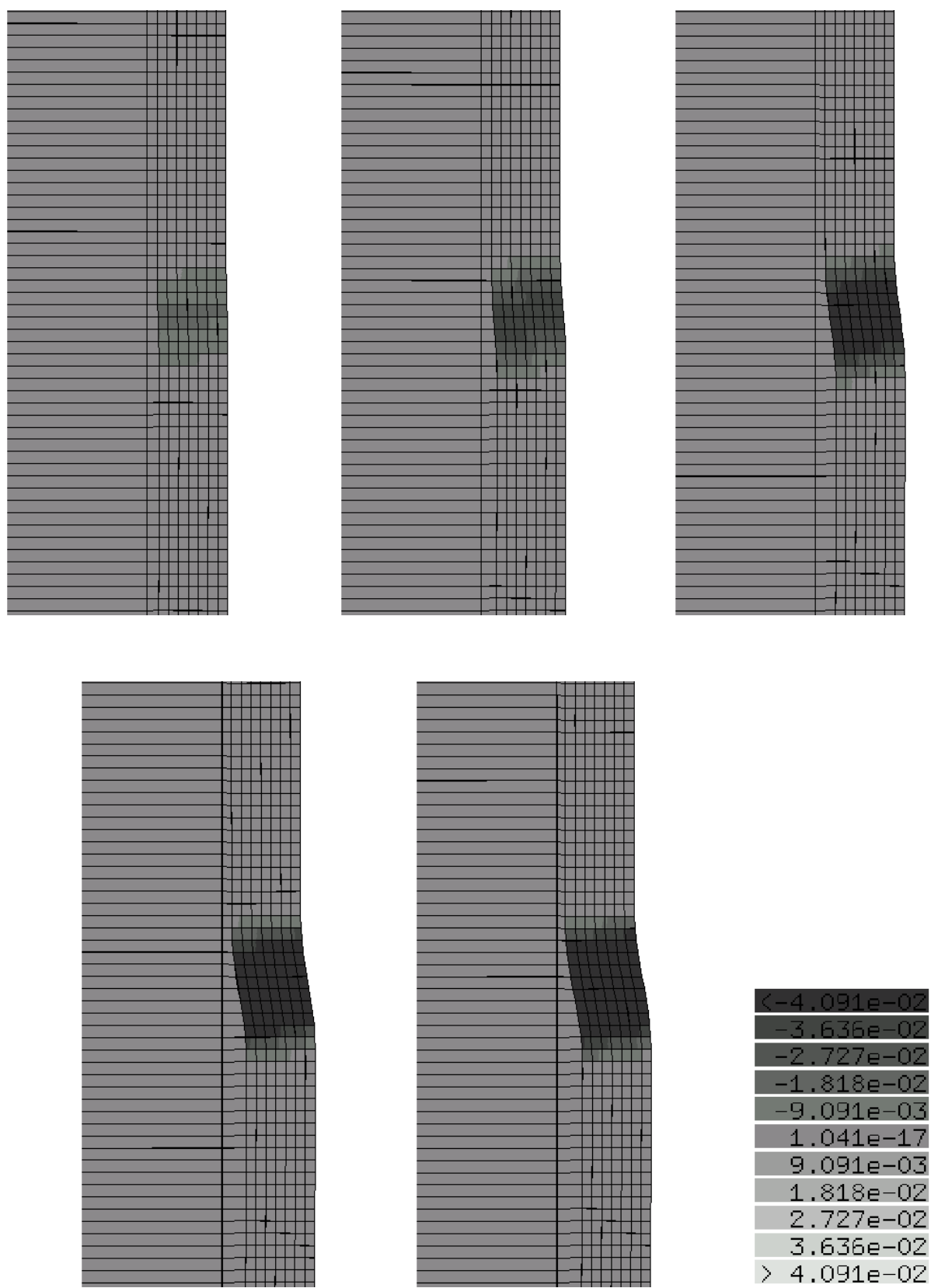

Figure 6: Distribution of $\gamma_{2}$ at the imperfection area of the deformed specimen. The range of the color map of the contours is fixed at $<-0.05 ; 0.05\rangle$. (Displacement in the $x$ direction magnified 10 times.) 



Figure 7: Distribution of $\tau_{2}$ at the imperfection area of the deformed specimen. The range of the contours is fixed at $<-31 ; 31>\mathrm{MPa}$. (Displacement in the $x$ direction magnified 10 times.) 


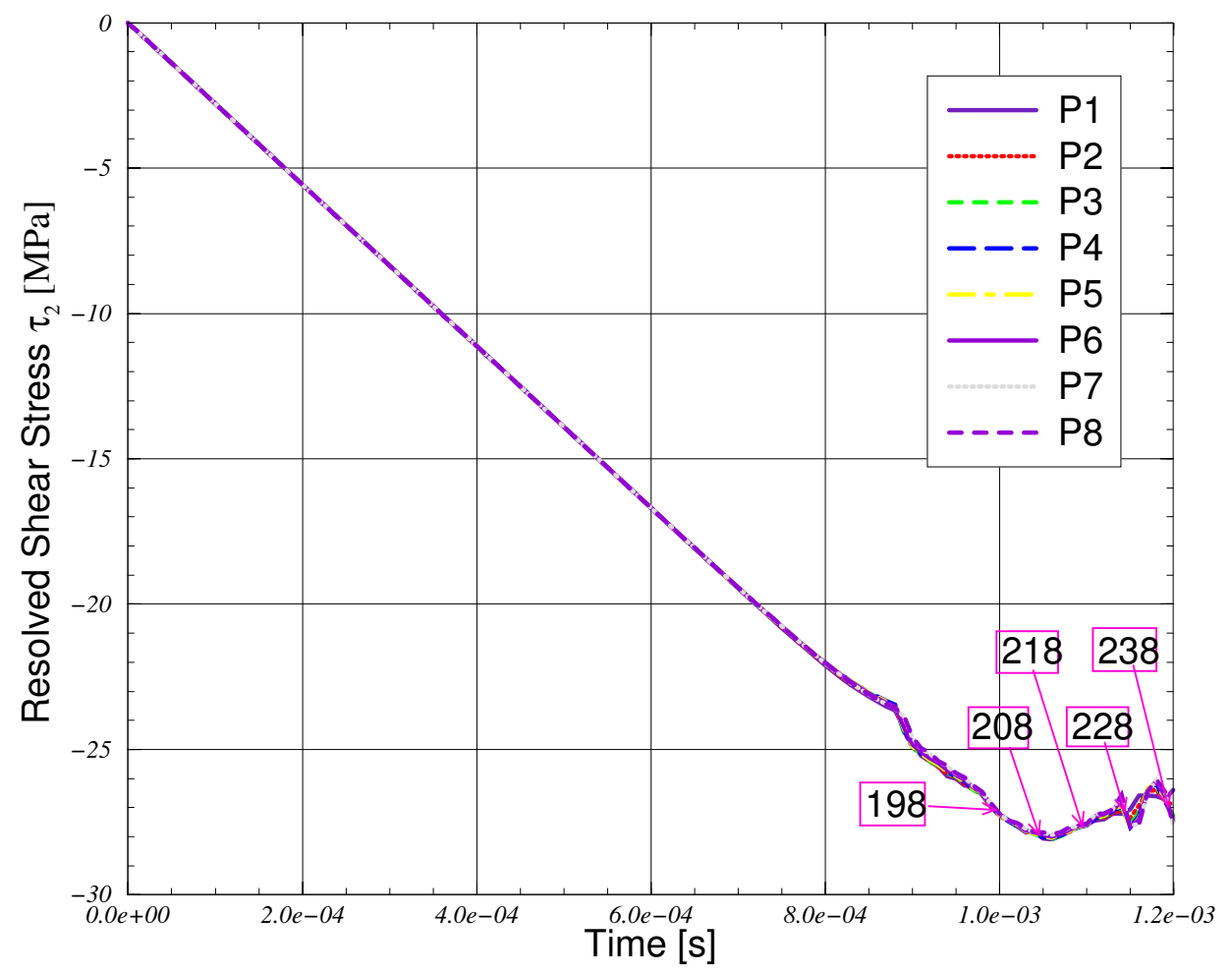

Figure 8: Resolved shear stress at the nodes on sampling line $(z=50 \mathrm{~mm})$ 


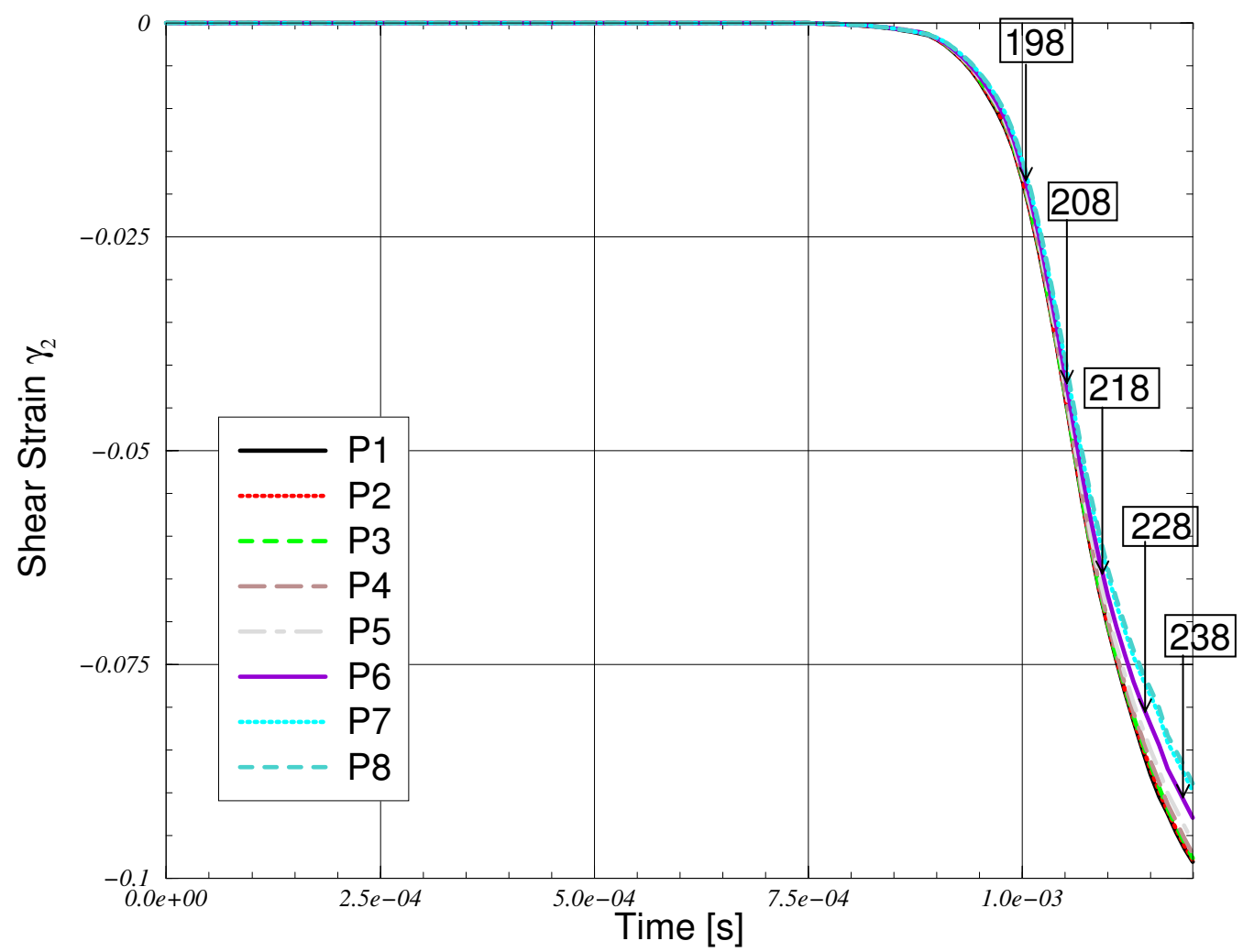

Figure 9: Shear strain $\gamma_{2}$ at the nodes on sampling line $(z=50 \mathrm{~mm})$ 


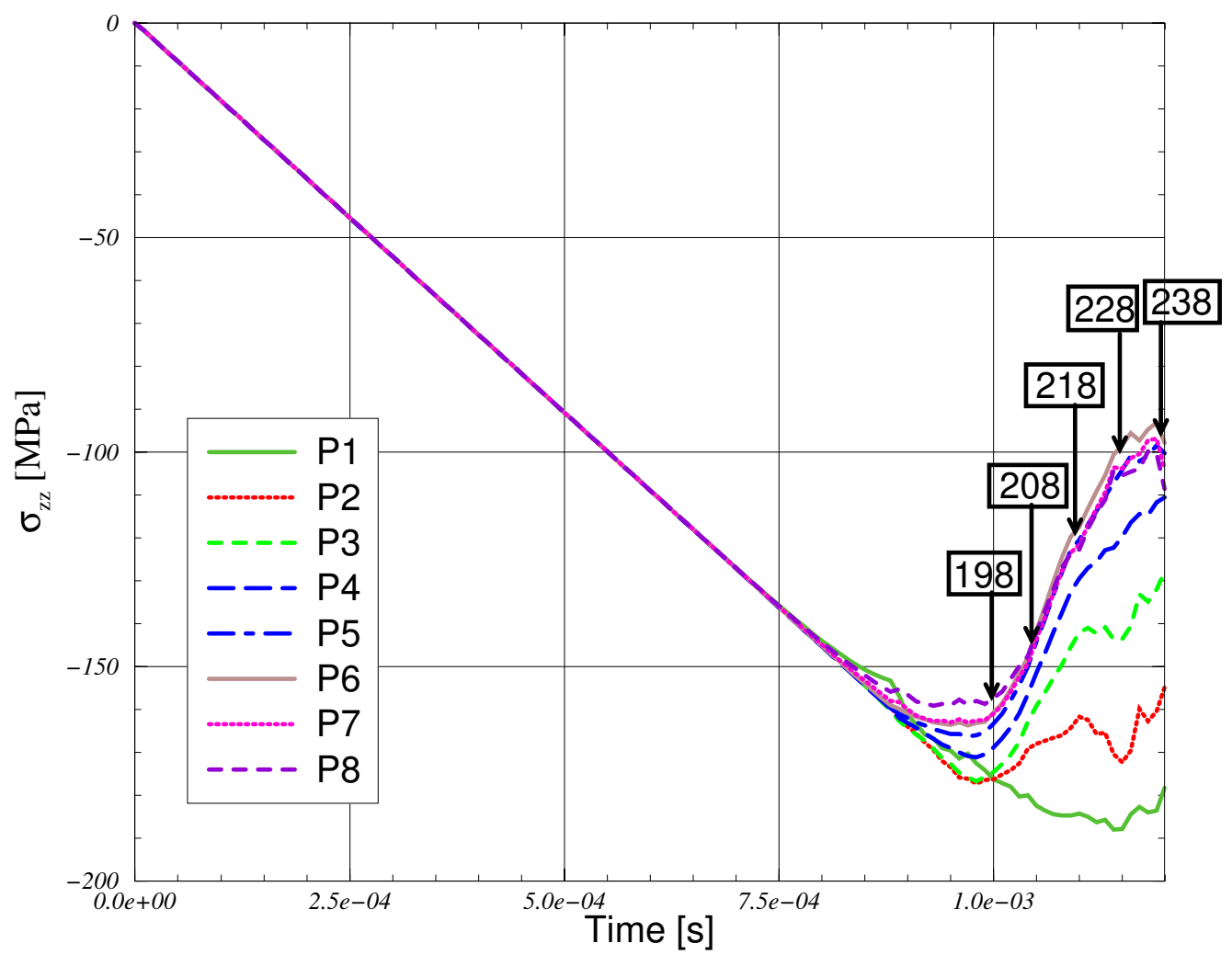

Figure 10: $\sigma_{z z}$ at the nodes on sampling line along fibers $(z=50 \mathrm{~mm})$ 


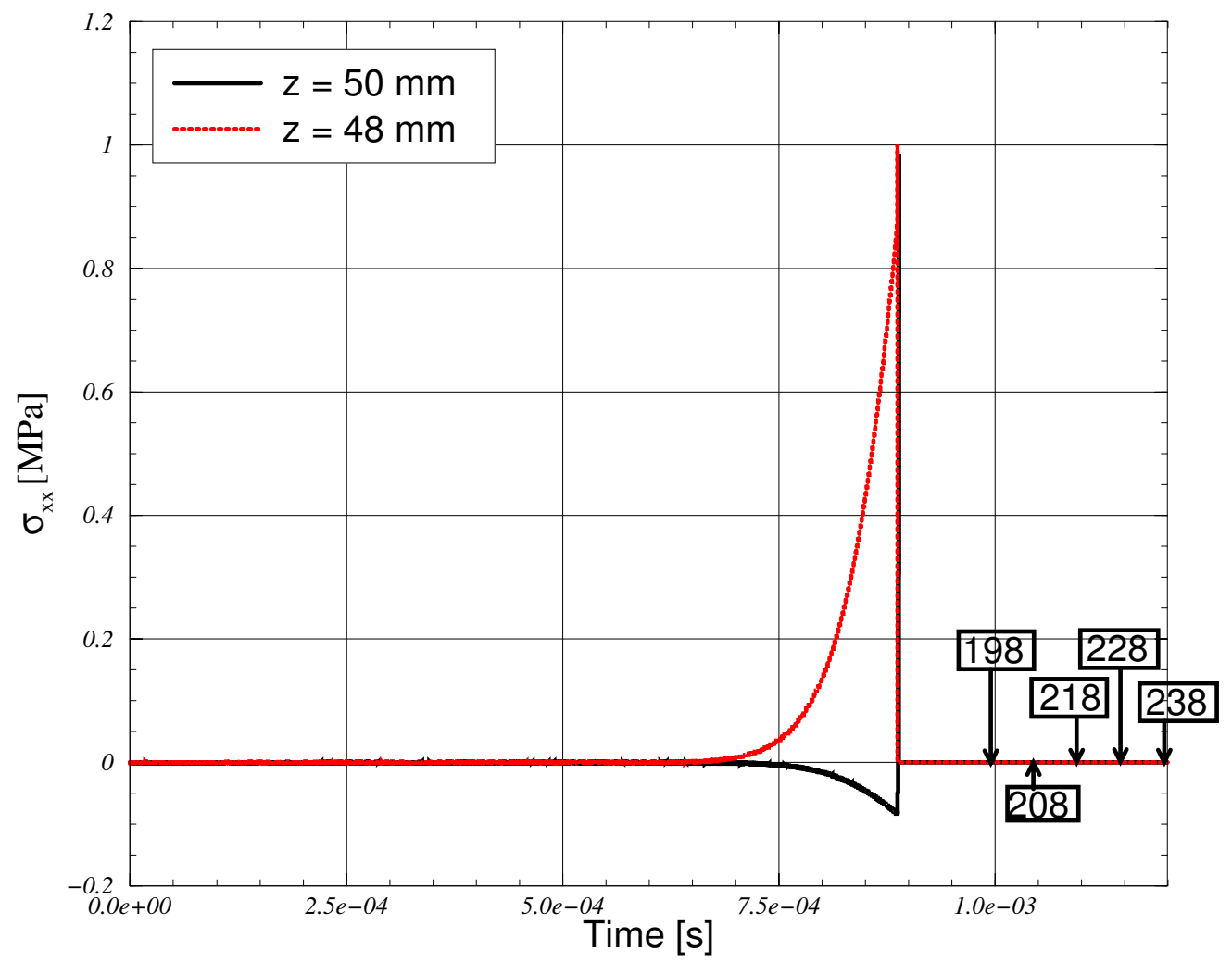

Figure 11: $\sigma_{x x}$ at the adhesive layer 


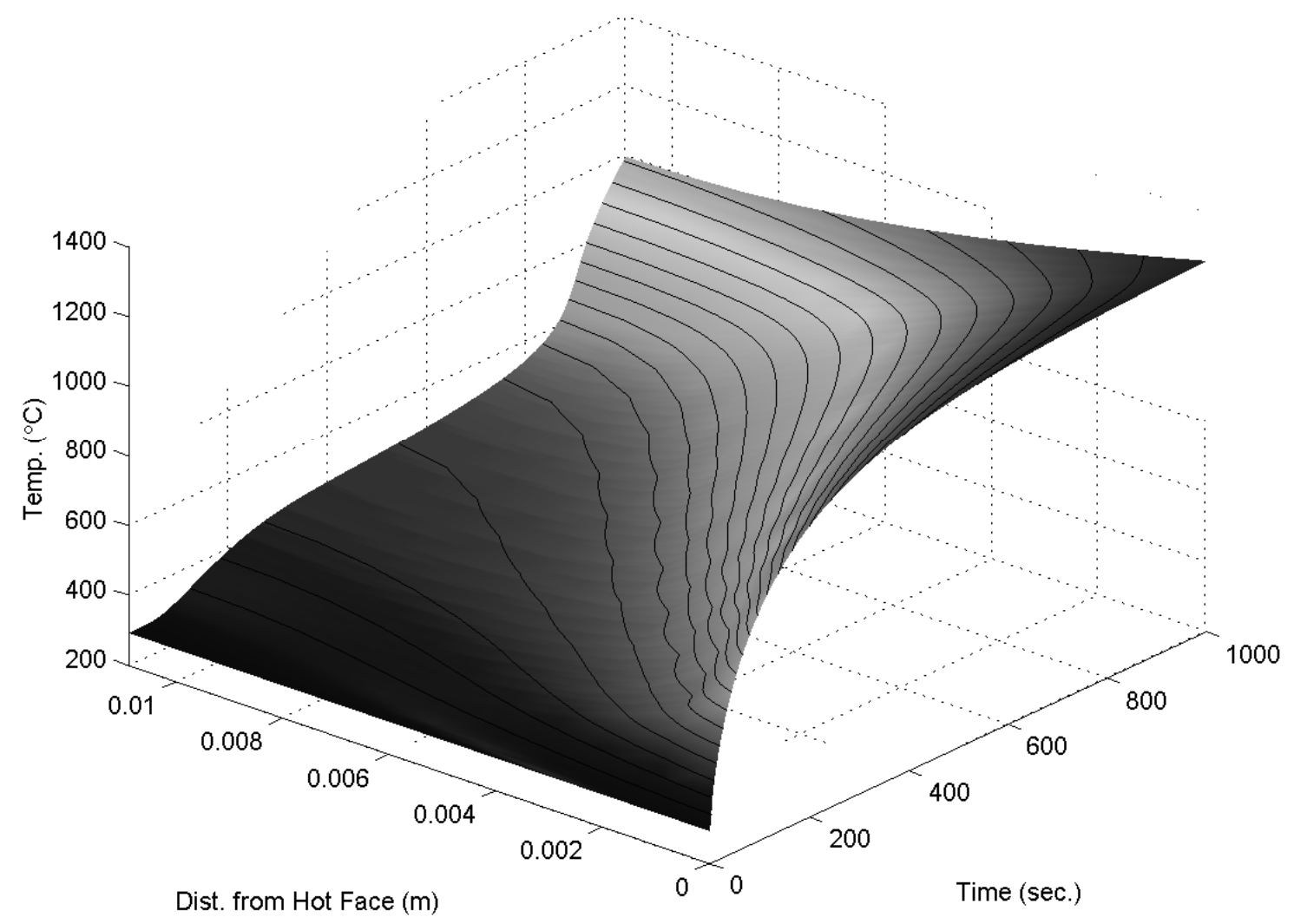

Figure 12: Thermal evolution of composite panel exposed to fire 